\title{
Mudanças organizacionais na adoção de tecnologias avançadas de manufatura
}

\author{
Rafaela da Rosa Cardoso, ${ }^{a, *}$, Edson Pinheiro de Lima \\ Sérgio Eduardo Gouvêa da Costa ${ }^{\mathrm{c}}$ \\ a,*rafaela.rosa@pucpr.br, PUCPR, Brasil \\ be.pinheiro@pucpr.br, PPGEPS/PUCPR, UTFPR, Brasil \\ 's.gouvea@pucpr.br, PPGEPS/PUCPR, UTFPR, Brasil
}

\begin{abstract}
Resumo
Para que a adoção de Tecnologia Avançada da Manufatura ( $A M T$ ) possa, de fato, proporcionar vantagens competitivas às empresas, faz-se necessário que essas tecnologias sejam selecionadas segundo critérios que considerem aspectos estratégicos. As características organizacionais de uma empresa influenciam sobremaneira o processo de adoção de $A M T$, de tal forma que se faz necessário desenvolver recomendações para a revisão do projeto organizacional. 0 presente trabalho propõe um processo para a geração de tais recomendações, através de uma metodologia denominada de "refinamentos sucessivos". Como resultado deste processo de "refinamentos sucessivos" obtém-se um conjunto de recomendações para a revisão do projeto organizacional, baseados em requisitos estabelecidos pelo processo de adoção de $A M T$. Essas recomendações constituem um conjunto de decisões a serem tomadas acerca do projeto organizacional para adequá-lo às demandas de uma nova tecnologia a ser introduzida, ou seja, a implementação de $A M T$.
\end{abstract}

Palavras-chave

Tecnologias avançadas de manufatura. Mudança organizacional. Estratégia de manufatura. Projeto organizacional.

\section{Introdução}

0 processo de introdução de uma nova tecnologia nas organizações produz mudanças na estrutura, processos e espaços organizacionais e está sujeito a barreiras no que se refere a sua implementação. Todos esses elementos relacionam-se a um novo desenho ou arquitetura organizacional, de tal forma que essas mudanças podem ser remetidas às revisões do projeto organizacional. Este artigo trata especificamente dos AMT(Advanced Manufacturing Technology - Tecnologia Avançada de Manufatura), que constituem o objeto da mudança, ou seja, a sua adoção e/ou implantação implica em mudanças na estrutura organizacional. Tais tecnologias são recursos mobilizados pelas empresas com o objetivo de desenvolver maiores níveis de competitividade. $\mathrm{Na}$ prática, no entanto, os $A M T$ são escolhidos segundo critérios predominantemente operacionais, visando, por exemplo, resolver problemas de qualidade, produtividade, segurança e de confiabilidade. Para que o emprego do $A M T$ possa, de fato, proporcionar vantagens competitivas às empresas, é necessário que as tecnologias sejam selecionadas segundo critérios que também considerem aspectos estratégicos (ABDEL-KADER; LUTHER, 2008; BROWN; BLACKMON, 2005).

Quando as organizações buscam a integração dos seus sistemas de produção, via automação, por exemplo, deparam-se com um problema: a concepção de um modelo ou a identificação de uma teoria para o projeto organizacional que seja compatível com as capacitações tecnológicas disponíveis e as demandas do ambiente externo ou mercado. A disponibilidade de novas tecnologias, como os $A M T$, e a consequente possibilidade de seu uso como uma arma para obter-se vantagem competitiva fazem com que as organizações, motivadas pela 
atualização tecnológica, reestruturem seus sistemas de operações. Tanto nos estudos acerca da inovação tecnológica como nos que avaliam especificamente a introdução de novas tecnologias, destaca-se a importância da compatibilidade da estrutura ou projeto organizacional com a tecnologia que se quer introduzir (DANGAYACH; DESHMUKH, 2005, 2006; HOFMANN; ORR, 2005; DÍAZ; MACHUCA; ÁlVAREZ-GIL, 2003; GHANI; JAYABALAN; SUGUMAR, 2002; BURCHER; LEE; SOHAL, 1999; DUIMERING; SAFAYENI; PURDY, 1993).

Baseando-se no modelo de seleção de $A M T$ desenvolvido por Gouvêa da Costa (2004), apresentado na Figura 1, o presente artigo busca identificar e verificar quais características organizacionais mediam o processo de seleção de $A M T$, de tal maneira que esse conhecimento possa subsidiar o processo de mudança organizacional, ou seja, a revisão do projeto organizacional.

Tendo em vista que a pesquisa apresentada neste artigo situa-se nos domínios da gestão de operações e da engenharia de produção, o enfoque qualitativo se apresenta como sendo o mais apropriado, pelas próprias características, para investigar as relações entre organização e tecnologia. A estratégia de pesquisa adotada fundamenta-se na construção de um quadro teórico, na utilização de dados secundários, na realização de entrevistas com especialistas e em estudos de caso. 0 processo adota uma abordagem de refinamentos sucessivos que culmina na identificação de um conjunto de

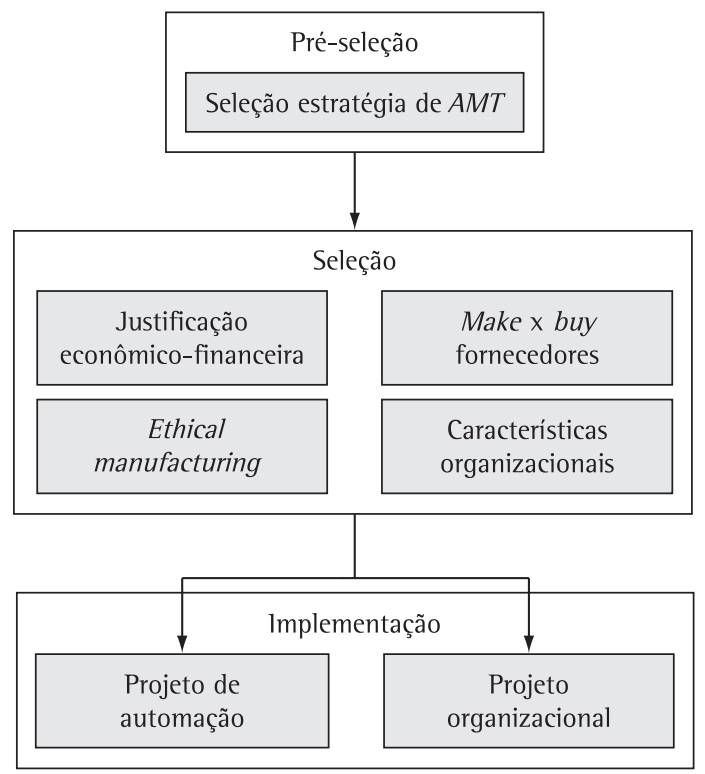

Figura 1. Modelo para seleção de $A M T$. Fonte: adaptado de Gouvêa da Costa (2004). características organizacionais que intervêm na adoção de $A M T$. 0 quadro-síntese refinado, ou seja, o produto gerado ao longo desta pesquisa, baseia-se em pesquisas já realizadas pelos próprios autores e em estudos publicados na literatura.

Tendo em vista a crescente busca pela utilização de $A M T$ nas organizações como meio de integração de seus sistemas, e como consequência as implicações em mudanças na estrutura organizacional, é que se apresenta a abordagem metodológica para identificar e verificar quais características organizacionais mediam o processo de seleção de tais tecnologias, de forma a minimizar as barreiras durante o processo de revisão do projeto organizacional. Para tanto serão apresentados o processo de "refinamentos sucessivos" utilizado, o referencial teórico que deu toda a sustentação ao desenvolvimento dos quadros-síntese mostrados e a lista de recomendações, ou seja, um conjunto de decisões a serem tomadas a partir de várias perspectivas, de modo a definir ou conduzir os impactos da adoção da tecnologia na organização.

\section{Desenvolvimento da pesquisa}

Para alcançar o objetivo de gerar as recomendações para a revisão do projeto organizacional, o trabalho baseou-se numa abordagem de pesquisa de natureza exploratória. Nesse sentido, partiu-se de um estudo primeiramente teórico, uma pesquisa bibliográfica, tendo como objetivo gerar uma lista de referência das recomendações de mudança organizacional, para que na sequência ela fosse refinada em cada etapa da pesquisa, através de um processo que se denominou de "refinamentos sucessivos", baseado na triangulação de dados e no cruzamento das recomendações com fontes de dados secundárias, como por exemplo pesquisas correlatas, entrevistas com profissionais e estudos de caso realizados. Como resultado desse processo obteve-se uma lista de recomendações para uma revisão refinada do projeto organizacional. Os resultados dessas etapas do processo de "refinamento sucessivo" serão apresentados na sequência.

Para viabilizar a estratégia de pesquisa, foram idealizadas quatro etapas, as quais são detalhadas a seguir. Partindo-se da revisão de literatura para a construção do corpo teórico do trabalho, foi possível identificar as características organizacionais e listar os principais problemas/ recomendações observados no processo de seleção/ adoção/implementação de $A M T$. Como resultado desta etapa, foi gerada uma lista de referência das recomendações. 
De posse desta lista foi realizado o primeiro refinamento, onde se cruzaram às recomendações, os dados de pesquisas correlatas. Essas fontes de dados secundários são constituídas pelos seguintes projetos: "Um estudo das 'barreiras' organizacionais à introdução de novas tecnologias" (CARDOSO; GOUVÊA DA COSTA; PINHEIRO DE LIMA, 2004) e "Sondagem industrial", desenvolvido pela FIEP (FEDERAÇÃO..., 2003，2004，2005，2006, 2007). Obteve-se, então, uma segunda lista de recomendações, resultado do primeiro refinamento.

0 segundo refinamento fundamenta-se em entrevistas realizadas com profissionais que participaram dos processos de seleção, adoção ou implementação de $A M T$ em empresas pertencentes ao setor automobilístico e metal-mecânico, gerando como resultado uma terceira lista de recomendações. Foram entrevistados oito profissionais das áreas de engenharia e de manufatura, distribuídos em três níveis hierárquicos (estratégico, gerencial e operacional), conforme se apresenta no Quadro 1. As entrevistas tiveram duração média de uma hora, seguindo um roteiro de entrevista semiestruturada baseando-se no relato das experiências dos entrevistados e na análise de documentação de projeto das empresas às quais pertenciam os entrevistados.

Onde AE significa "Entrevistado A", BE significa "Entrevistado B" e assim sucessivamente.

0 terceiro refinamento foi realizado a partir da análise dos dados coletados de três estudos de caso desenvolvidos em empresas do setor automobilístico, metal-mecânico e madeireiro. Dada a natureza exploratória do estudo, optou-se por analisar empresas pertencentes a indústrias diferentes, mas que representassem setores com alto grau de adoção de $A M T$. Tais empresas pertencem a setores com elevado grau de modernização tecnológica, conforme evidenciado na análise de competitividade realizada pelo ECIB (BRASIL, 1993) e por informações obtidas junto a integradores.

A lista de recomendações obtida ao final deste terceiro refinamento é apresentada como uma lista base para um processo de teste através da realização de um survey, que não consta desta pesquisa, mas constitui o seu desdobramento futuro. 0 Quadro 2 apresenta um resumo das evidências coletadas junto aos estudos de caso, onde foram trabalhadas algumas palavras-chave para busca em alguns tipos de documentos (como manual da qualidade, organograma, projeto de implantação).

Os estudos de caso foram conduzidos da seguinte forma: um primeiro contato para definir quais pessoas estariam envolvidas nas entrevistas e uma visita à planta/instalações da empresa para melhor entender o seu sistema de produção. As entrevistas foram inicialmente organizadas em duas partes, uma para apresentar o projeto, criar um vocabulário comum e identificar a visão do entrevistado frente à introdução da tecnologia que está sendo analisada; e uma segunda para aplicar um questionário com perguntas objetivas visando identificar os benefícios/problemas decorrentes dos processos de seleção, adoção ou implementação de AMT.

No decorrer da aplicação dos estudos de caso observou-se que o tempo para sua realização estava se estendendo demais, devido à agenda dos participantes das empresas. Como forma de minimizar esse fato, as entrevistas passaram a ser realizadas num único momento. As duas partes que antes eram feitas em meia hora aproximadamente, cada uma, foram dimensionadas para reuniões de uma hora para uma aplicação única de todo o processo de entrevista.

Quadro 1. Dados dos entrevistados.

\begin{tabular}{|c|c|c|c|}
\hline \multirow{2}{*}{ Entrevistados } & \multicolumn{2}{|c|}{ Área de atuação } & \multirow{2}{*}{ Formação acadêmica dos entrevistados } \\
\cline { 2 - 4 } & Engenharia & Manufatura & $\begin{array}{c}\text { Engenheiro Eletrônico e Mestre em } \\
\text { Engenharia Elétrica e lnformática Industrial }\end{array}$ \\
\hline AE & Engenheiro de Projeto & & Engenheiro Eletricista \\
\hline BE & Gerente de Projetos & & Engenheiro Mecânico \\
\hline CE & Gerente de Engenharia & & Engenharia de Produção Mecânica \\
\hline DE & & & Engenheiro Eletricista \\
\hline FE & Supervisor de Linha & Gerente de planta & $\begin{array}{c}\text { Engenheiro Mecânico e Especialista em } \\
\text { Gestão Estratégica de Negócios }\end{array}$ \\
\hline GE & Engenheiro de Projeto & & $\begin{array}{c}\text { Tecnólogo em Processamento de Dados e } \\
\text { Mestre em Engenharia de Produção e Sistemas }\end{array}$ \\
\hline HE & & Operador de máquina & Técnico em Eletrônica \\
\hline
\end{tabular}

Fonte: elaborado pelos autores. 
Quadro 2. Dados dos estudos de caso.

\begin{tabular}{|c|c|c|c|c|c|}
\hline & & & \multicolumn{3}{|c|}{ Empresa } \\
\hline \multicolumn{3}{|c|}{ Fase 1} & $x$ & Y & $\mathrm{Z}$ \\
\hline \multirow[t]{4}{*}{ Entrevista } & Área & Nível & \multicolumn{3}{|c|}{ Tempo de duração (horas) } \\
\hline & Eng. de projeto & Gerencial & 1.0 & 1.0 & 1.0 \\
\hline & Manufatura & Operacional & 0.5 & 0.5 & 1.0 \\
\hline & Manufatura & Gerencial & 1.0 & 0.5 & 1.0 \\
\hline \multicolumn{3}{|c|}{ Fase 2} & $x$ & Y & Z \\
\hline \multirow[t]{4}{*}{ Análise documental } & \multicolumn{2}{|c|}{ Tipo de documento } & \multicolumn{3}{|c|}{ Tempo de duração (horas) } \\
\hline & \multicolumn{2}{|c|}{ Organograma } & 0.25 & 0.25 & 0.25 \\
\hline & \multicolumn{2}{|c|}{ Manual de qualidade } & 0.5 & 0.5 & 0.5 \\
\hline & \multicolumn{2}{|c|}{ Projeto de implantação } & 0.5 & 0.5 & 0.5 \\
\hline \multicolumn{3}{|c|}{ Fase 3} & $\mathrm{x}$ & Y & Z \\
\hline \multirow[t]{2}{*}{ Visita técnica } & \multicolumn{2}{|c|}{ Tipo de processo } & Em linha & Job shop & Em linha \\
\hline & \multicolumn{2}{|c|}{ Grau de automação } & Automatizado & Automatizado & Semi-automatizado \\
\hline \multicolumn{3}{|c|}{ Início } & $27 / 07 / 2005$ & $07 / 10 / 2005$ & $05 / 11 / 2005$ \\
\hline \multicolumn{3}{|c|}{ Conclusão } & $19 / 09 / 2005$ & 05/01/2006 & $11 / 2 / 2006$ \\
\hline \multicolumn{3}{|c|}{ Descrição da empresa } & $\begin{array}{c}\text { Setor } \\
\text { automobilístico } \\
\text { de grande porte }\end{array}$ & $\begin{array}{l}\text { Fornecedora direta do } \\
\text { setor automobilístico } \\
\text { de grande porte }\end{array}$ & $\begin{array}{l}\text { Ramo madeireiro } \\
\text { de grande porte }\end{array}$ \\
\hline
\end{tabular}

Fonte: elaborado pelos autores.

A Figura 2 esclarece a delimitação ou "funil" que ocorreu na pesquisa. Partiu-se de uma análise mais ampla baseada no framework para o processo de seleção de $A M T$, buscando na literatura informações a respeito dos principais problemas/ recomendações observados no processos de seleção, adoção ou implementação de $A M T$. Na sequência foram analisados trabalhos correlatos, depois as entrevistas com profissionais (especialistas), para então compilar as recomendações com base nos resultados de três estudos de caso.

Tecidas as considerações iniciais a respeito dos procedimentos metodológicos adotados, podem-se apresentar em maior detalhe os resultados obtidos pelos "refinamentos sucessivos".

\section{Desenvolvimento das recomendações}

0 desenvolvimento das recomendações tem como ponto de partida a construção do referencial teórico, para então aplicar o processo de "refinamentos sucessivos", usando para tanto informações obtidas junto a pesquisas correlatas, entrevistas com especialistas e estudos de caso.

\subsection{Da construção do referencial teórico}

No framework proposto por Gouvêa da Costa, Platts e Fleury (2006), apresentado na Figura 3, os $A M T$ são entendidos como recursos e têm por objetivo formar determinadas capacitações. Tais capacitações encontram-se declaradas na "visão

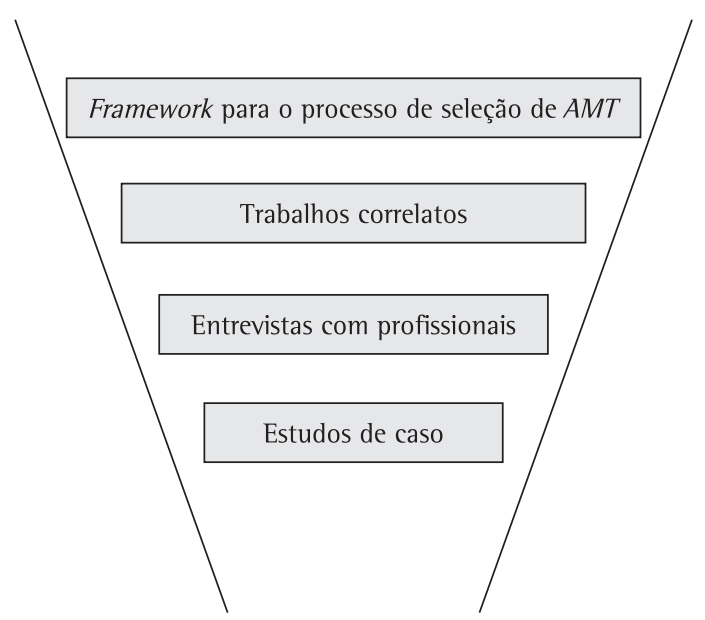

Figura 2. Delimitação da pesquisa. Fonte: elaborado pelos autores.

da manufatura", que é a descrição do conjunto de capacitações relacionadas ao sistema de manufatura que o negócio espera desenvolver.

0 processo de seleção de $A M T$, descrito na Figura 1, envolve aspectos estratégicos, aspetos da ética na manufatura, a justificação econômicofinanceira dos $A M T$, relação com fornecedores e o processo de implantação dos $A M T$ na organização. Particularmente, posiciona-se o presente trabalho na fase de implantação, na medida em que o reprojeto organizacional prepara a empresa para as mudanças advindas da adoção de um $A M T$ (GOUVÊA DA COSTA, 2004). 


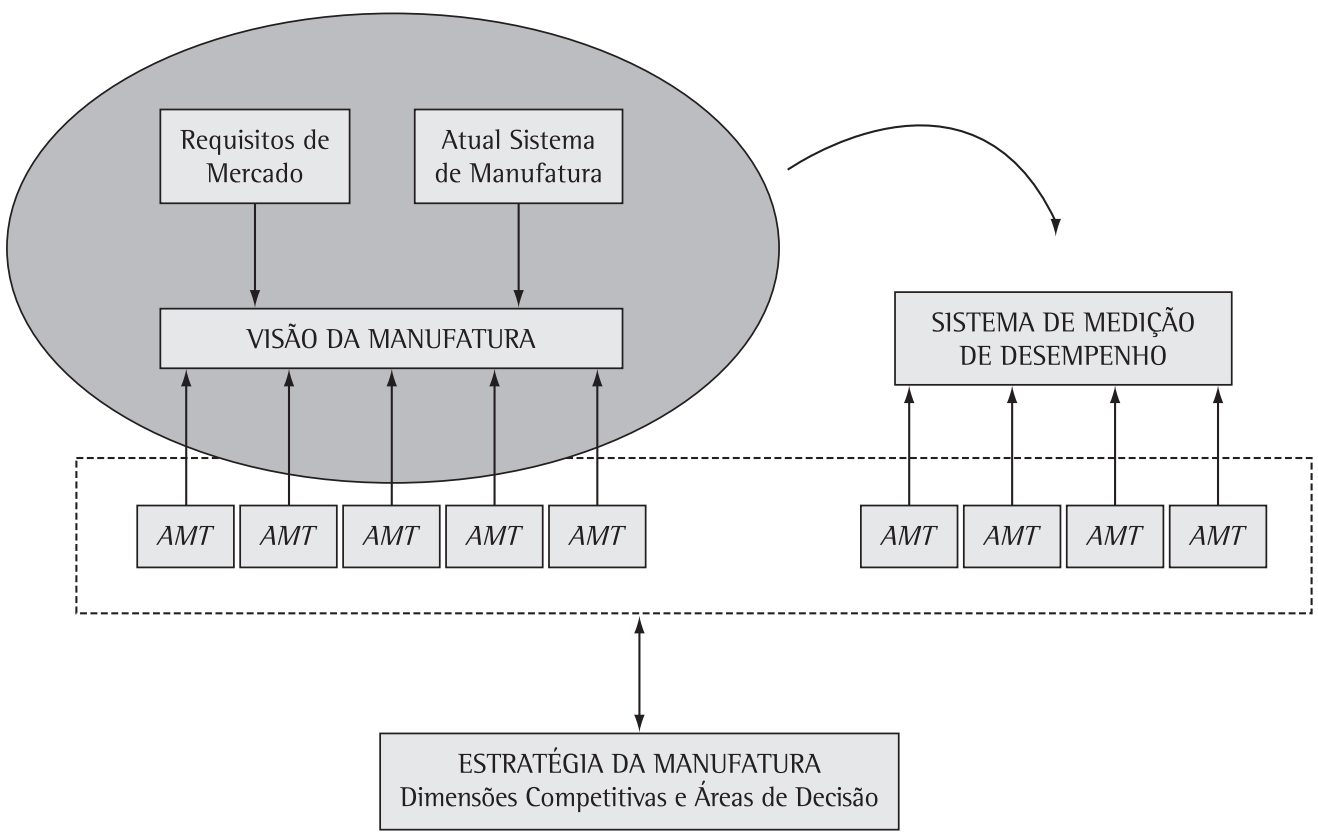

Figura 3. Framework para o processo de seleção de AMT. Fonte: Gouvêa da Costa, Platts e Fleury (2006).

A análise prévia do "impacto" na adoção e na implementação de um $A M T$ na organização contribui para o processo de seleção de $A M T$, como pode ser observado no framework da Figura 1.

Algumas das questões que podem ser antecipadas no sentido de minimizar, ou até mesmo eliminar, as barreiras à introdução de $A M T$ podem ser declaradas da seguinte forma:

- Quais são as implicações, em termos de alterações organizacionais, decorrentes da adoção ou implementação de $A M T$ ?

- Um determinado $A M T$ determina um conjunto específico de características organizacionais? 0 inverso também ocorre?

- Qual opção de $A M T$ implica em maior compatibilidade com aspectos organizacionais (estrutura, processos, espaços, cultura organizacional etc.)?

Como forma de minimizar esse impacto, a gestão da mudança envolve a compreensão do contexto organizacional, assim como a capacidade de agir e mobilizar recursos para promovê-la (MARQUES; CHAVES; DIAS, 2005). Segundo Marques, Borges e Carvalho-Freitas (2007), quando se trata de gestão da mudança dentro das organizações o maior obstáculo ao sucesso da introdução de mudanças organizacionais é a resistência a elas, tanto por fatores individuais quanto situacionais no processo. Que, segundo Maravieski e Reis (2008), é necessário planejar a mudança para que as resistências sejam minimizadas.
No caso deste estudo em particular, será abordado o processo da introdução dos $A M T$ na organização, em específico o estudo das características organizacionais. Para tanto existem na literatura trabalhos direcionados a esse tipo de tecnologia, como apresentado na sequência do artigo. E para realizar o estudo acerca das características organizacionais e sua relação com os $A M T$, respondendo às questões propostas, utilizou-se de um framework que representasse as dimensões tratadas no projeto organizacional, ou seja, “domínios" para localizar as contribuições.

0 framework de referência para o projeto organizacional tem como premissa o estudo da forma (aspectos estruturais). Está organizado em áreas de definição ou de estudo, ou seja, constitui um sistema de aspectos (CHURCHMAN, 1972). Dessa maneira, pode-se estudar a organização na medida da sua estrutura, que define os mecanismos de coordenação e o tipo de orientação a ser dado às relações internas e externas; ou de seus processos que representam a própria ação organizacional; ou no âmbito dos seus "espaços" que dão "lugar" às interações humanas para "criar" e "participar". 0 papel do framework apresentado na Figura 4 é o de organizar a análise a ser desenvolvida no âmbito deste trabalho (PINHEIRO DE LIMA; LEZANA, 2005).

0 contexto deste framework é fundamentado numa concepção de estratégia baseada em competências (competências requeridas). Assim 


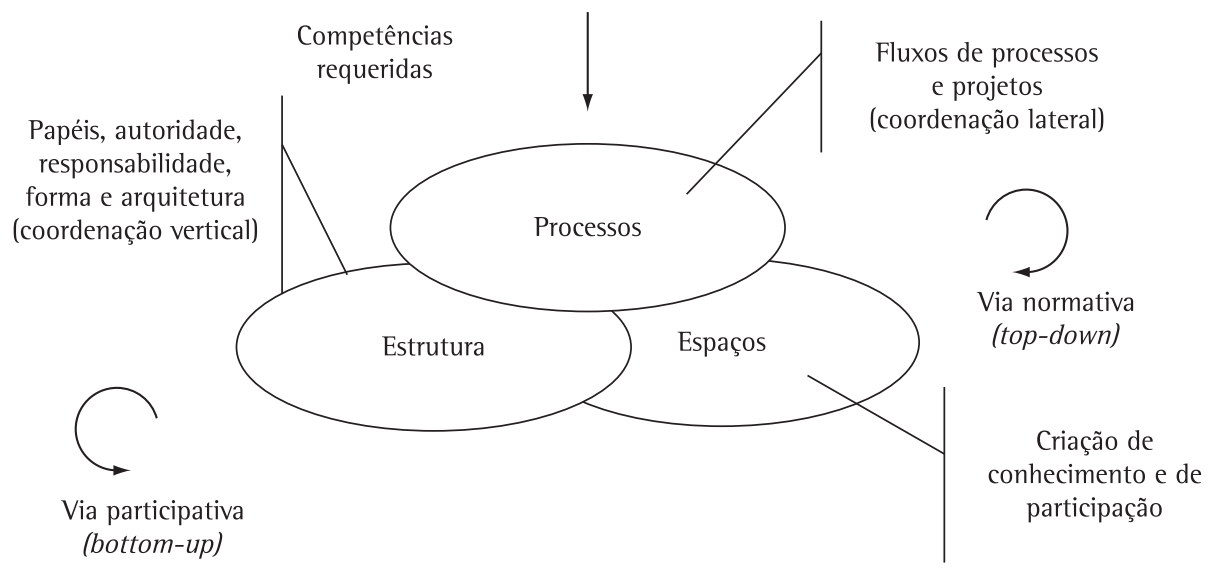

Figura 4. Framework para o projeto organizacional. Fonte: Pinheiro de Lima e Lezana (2005).

sendo, o projeto organizacional se orienta na criação de um conjunto de condições necessárias, nas suas diferentes dimensões, para que tais competências sejam desenvolvidas pela empresa. Os AMT constituem recursos a serem mobilizados em "competências" que definem a visão da manufatura. Sendo assim, a abordagem centrada em competências e na visão da manufatura estabelece a "ponte" para se estudar a relação entre $A M T$ e o projeto organizacional (GOUVÊA DA COSTA; PINHEIRO DE LIMA, 2009).

As informações levantadas na revisão teórica, nos trabalhos correlatos, nas entrevistas e nos estudos de caso foram organizadas de acordo com as dimensões estabelecidas pelo framework do projeto organizacional, ou seja, de acordo com as dimensões: estrutura, processos e espaços (Figura 4).

Conforme o proposto pela metodologia dos "refinamentos sucessivos", ao final de cada etapa de refinamento foi gerado um quadro-síntese com suas respectivas observações. 0 quadro-base foi gerado a partir da revisão da literatura. Neste quadro estão apresentadas em síntese as contribuições da literatura, no sentido de organizar os problemas/ recomendações observados nos processos de seleção, adoção ou implementação de $A M T$, de acordo com as dimensões relativas ao framework do projeto organizacional. 0 Apêndice 1 representa o "quadro-base" organizado de acordo com as dimensões: estrutura, processos e espaços.

Definidos os elementos organizacionais na dimensão estrutura, pode-se organizar a análise em termos de processos. É importante destacar as relações de interdependência que se estabelecem entre eles via projeto organizacional. Pode-se tratar das relações entre recursos, em particular $A M T$, e estrutura na medida da sua alocação, enquanto a dimensão processos define elementos para a sua mobilização.
As dimensões estrutura e processos associam-se aos espaços organizacionais por intermédio do posicionamento do $A M T$, ou seja, da definição de seu locus, que em última instância define a sua utilidade e valor. Sendo estas definidas em termos de mercado ou nas relações interpessoais.

Dado o referencial teórico, representado por uma lista de "recomendações" para o projeto organizacional quando da adoção de $A M T$, pode-se partir para o processo de "refinamentos sucessivos". Dadas as restrições de formatação e conteúdo do artigo, apenas será apresentada a versão final da lista de recomendações, no entanto são destacadas as principais modificações oriundas dos refinamentos.

\subsection{Das fontes de dados secundários}

0 primeiro refinamento resultou num quadro com problemas/recomendações asseguradas pelo projeto "Um estudo das 'barreiras' organizacionais à introdução de novas tecnologias" e pela "Sondagem industrial" da FIEP. Para cada uma das dimensões foram identificadas as recomendações/problemas de relevância também para esses estudos. Essa relevância é identificada através de um processo de filtragem dos dados através do consenso identificado nas fontes. Dessa forma o quadro gerado após essa etapa foi assegurado por trabalhos de nível nacional.

No projeto "Um estudo das 'barreiras' organizacionais à introdução de novas tecnologias" foram realizados três estudos de caso com 29 entrevistados de vários níveis organizacionais, gerando um conjunto de recomendações que representam as maiores barreiras que se interpõem ao processo de introdução de uma nova tecnologia na organização. 
$\mathrm{Na}$ "Sondagem industrial" (pesquisa que capta as expectativas dos líderes empresariais do estado do Paraná com relação ao desenrolar de suas atividades no futuro próximo, bem como o que de concreto está sendo desenvolvido para fazer frente aos novos e constantes desafios), que vem sendo realizada desde 1996, surgiu a oportunidade de inserir duas questões de caráter tecnológico relacionadas aos benefícios e riscos oferecidos pela introdução de um AMT, a partir de 2003. Tem-se hoje um histórico de cinco anos dessas questões apresentado através das Figuras 5 e 6.

Verifica-se que nesses cinco anos de aplicação das questões sobre $A M T$, os pontos "aumento da qualidade" e "redução de custos" foram benefícios constantes observados pelas empresas. 0 fato de elas darem grande importância às questões de cunho econômico-financeiro também é confirmado por outras evidências neste trabalho. Em relação aos problemas observados, os pontos "dificuldade em atingir as metas financeiras" e "interrupção durante a implementação" foram listados em todas as pesquisas realizadas. Se as organizações selecionassem as tecnologias mais adequadas em conformidade com o framework para seleção de $A M T$ (Figura 1), é bem provável que não fossem verificadas interrupções durante a implantação com tanta frequência.

\subsection{Das entrevistas com especialistas}

Da análise dos dados coletadosnas entrevistas com profissionais foi gerado um quadro correspondente à segunda etapa de refinamento. Novamente foi aplicado um processo de filtragem dos dados através do consenso identificado nas fontes. Nessa etapa foram encontradas significativas reduções na quantidade de problemas/recomendações, pois a partir daí a pesquisa estava delimitada aos setores automobilístico, metal-mecânico e madeireiro.

Um dos pontos unânimes nas entrevistas realizadas foi a realocação dos funcionários, ou seja, com o projeto de implementação de um $A M T$ na organização houve preocupação com as pessoas envolvidas no processo. Em nenhuma das empresas foi necessária a demissão de pessoal devido à introdução do $A M T$. Muito pelo contrário, elas tiveram a oportunidade de passar por programas de capacitação para estarem aptas a trabalhar com a nova tecnologia. E percebendo essa preocupação por parte da organização, os funcionários se sentem parte do processo. De acordo com o Entrevistado E: “[...] preocupação com a mão-de-obra, com a cultura organizacional. O foco é no operador".
Principais benefícios associados com a introdução de AMT's

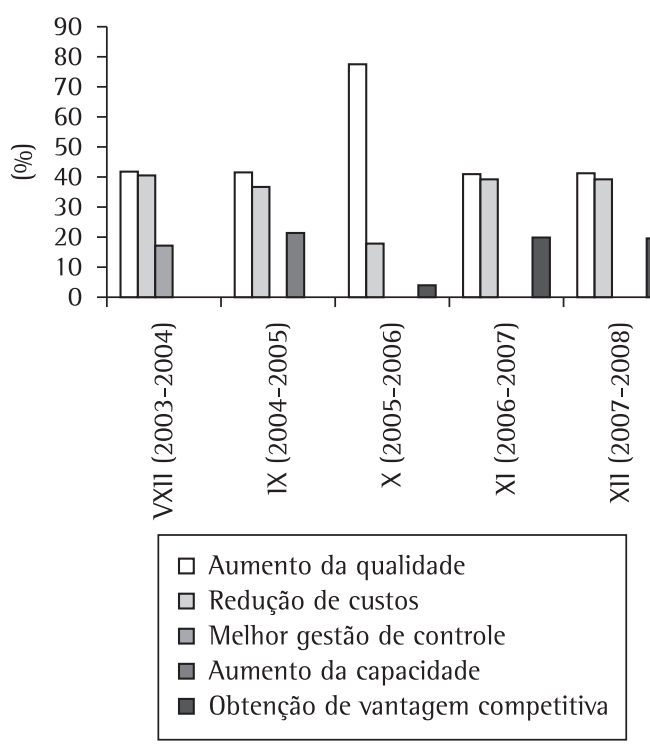

Figura 5. Histórico dos principais benefícios associados à introdução de $A M T$. Fonte: elaborado pelos autores.

Principais problemas verificados com a introdução de $A M T$ 's

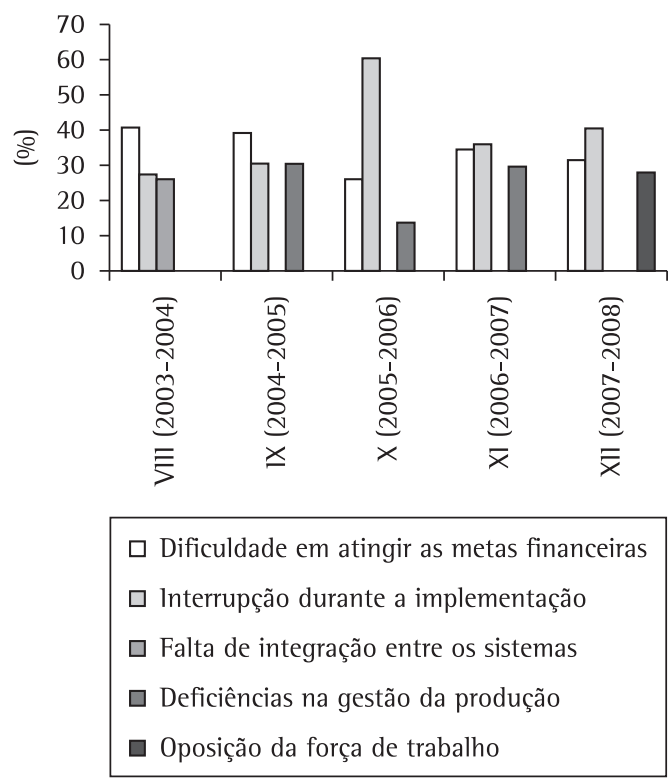

Figura 6. Histórico dos principais problemas associados à introdução de $A M T$. Fonte: elaborado pelos autores.

Outro ponto de consenso foi a criação de times de trabalho para o projeto de implementação de um $A M T$ na organização. As decisões ao longo do processo sempre foram tomadas por um grupo de gestores de várias áreas da empresa. 0 que é 
interessante pelo fato de vários pontos de vista e consequentemente várias perspectivas serem levadas em consideração.

\subsection{Dos estudos de caso}

0 terceiro refinamento foi obtido através da realização de três estudos de caso. As recomendações contidas nesse quadro foram citadas por pelo menos dois dos estudos de caso realizados. 0 último refinamento reforçou a lista de problemas/ recomendações gerado, tendo como base as análises realizadas nos estudos de caso, onde os dados foram compilados, analisados e sintetizados para gerar o quadro-síntese geral da pesquisa, representado aqui pelos Quadros 3, 4 e 5.

Observa-se do Quadro 3 que aspectos que envolvem a organização do trabalho, o desenvolvimento de uma componente gerencial e a formação de competências marcaram as principais recomendações em termos estruturais. Neste momento vale a pena destacar que existem dois problemas/recomendações - flexibilidade da organização e alinhamento estratégico - que não foram identificados como prioritários no decorrer dos "refinamentos sucessivos", mas que segundo o levantamento bibliográfico também são importantes no processo de mudança organizacional.
Do Quadro 4 identifica-se que o conhecimento das relações causa-efeito e o processo de gestão ou controle estratégico caracterizam as contribuições no nível processos. 0 problema/recomendação "modificação de processos e infraestrutura" seria outro exemplo que deveria passar por uma nova pesquisa, pois segundo referências - por exemplo, Das e Narasimhan (2001); Ghani, Jayabalan e Sugumar (2002); Díaz, Machuca e Álvarez-Gil (2003); Raymond e St-Pierre (2005) -, esse seria um ponto muito importante no processo de preparação para a adoção de um $A M T$.

Pode-se concluir que no Quadro 5 a componente sistêmica da abordagem de seleção, adoção e implementação de $A M T$ é evidenciada. Promove-se, portanto, uma síntese "sistêmica" do uso do recurso AMT.

A partir desses quadros verifica-se a importância das "áreas de decisão" do framework do projeto organizacional dentro do processo de seleção de AMT. As "recomendações organizacionais" são enquadradas nas "áreas de decisão", ou seja, nas dimensões, e através das relações entre elas desenvolve-se o modelo organizacional.

Estes são pontos-chave no processo de seleção de $A M T$, ou seja, a maioria das empresas estudadas (através dos relatos da literatura e dos estudos de caso) levou esses pontos em consideração a partir do momento em que decidiram introduzir uma nova tecnologia em seus processos.

Quadro 3. Quadro-síntese geral da pesquisa para a dimensão estrutura.

\begin{tabular}{|c|c|}
\hline Problema / Recomendação & \multirow{2}{*}{ Implicações para a revisão do projeto organizacional } \\
\hline Definição & \\
\hline $\begin{array}{l}\text { Grupo de coordenação } \\
\text { Definição de um grupo de coordenação pra gerir o } \\
\text { processo de seleção/adoção/ implementação de } A M T\end{array}$ & $\begin{array}{l}\text { As decisões ao longo do processo de implementação de um } A M T \text { normalmente } \\
\text { são tomadas por um grupo de gestores de diversas áreas da organização. } 0 \text { que é } \\
\text { interessante pelo fato de vários pontos de vista e consequentemente várias perspectivas } \\
\text { serem levadas em consideração }\end{array}$ \\
\hline $\begin{array}{l}\text { Times de trabalho } \\
\text { Criação de times de trabalho ao longo do processo }\end{array}$ & $\begin{array}{l}\text { Tendo em vista que as decisões são tomadas normalmente por gestores de diversas } \\
\text { áreas, a criação de times de trabalho torna-se ponto-base para que o desenvolvimento } \\
\text { do projeto ocorra da melhor maneira possível }\end{array}$ \\
\hline $\begin{array}{l}\text { Programas de capacitação } \\
\text { Realizar programas de capacitação para todos os } \\
\text { níveis relacionados à introdução do } A M T\end{array}$ & $\begin{array}{l}\text { A capacitação de todos os envolvidos no processo de seleção/adoção/implementação } \\
\text { de um } A M T \text { assegura que estão aptos a realizar as operações do processo }\end{array}$ \\
\hline
\end{tabular}

Fonte: elaborado pelos autores.

Quadro 4. Quadro-síntese geral da pesquisa para a dimensão processos.

\begin{tabular}{|l|l|}
\multicolumn{1}{|c|}{ Problema/Recomendação } & \multicolumn{1}{|c|}{ Implicações para a revisão do projeto organizacional } \\
\cline { 1 - 1 } Definição & Para a efetivação e desenvolvimento do processo, o acompanhamento deve ocorrer \\
Acompanhamento contínuo \\
$\begin{array}{l}\text { Realizar um acompanhamento continuo ao longo e permanentemente, garantindo que marcos de projeto sejam alcançados, e } \\
\text { do processo }\end{array}$
\end{tabular}

Fonte: elaborado pelos autores. 
Quadro 5. Quadro-síntese geral da pesquisa para a dimensão espaços.

\begin{tabular}{|c|c|}
\hline Problema/Recomendação & \multirow{2}{*}{ Implicações para a revisão do projeto organizacional } \\
\hline Definição & \\
\hline $\begin{array}{l}\text { Importância de "cada um" no todo } \\
\text { Mostrar a importância de cada um para que o todo } \\
\text { funcione da melhor maneira }\end{array}$ & $\begin{array}{l}\text { Tomando como exemplo o estudo de caso } X \text {, onde o supervisor da nova linha foi até a } \\
\text { matriz da empresa para um treinamento, ele sentiu como se fosse parte do processo, se } \\
\text { sentiu responsável por ele, tendo impacto direto no comprometimento e motivação da } \\
\text { equipe durante e após a implementação do projeto }\end{array}$ \\
\hline
\end{tabular}

Fonte: elaborado pelos autores.

\section{Algumas considerações acerca da pesquisa e dos resultados}

Da pesquisa realizada observa-se que os problemas verificados após a implementação de $A M T$ decorrem em grande parte da não análise, ou análise incompleta, das características organizacionais requeridas pelo processo de seleção de $A M T$.

Cabe salientar alguns pontos que durante a pesquisa de campo necessitaram de adaptações, pois, quando se realizam entrevistas ou estudos de caso, deve-se estar preparado para trabalhar com a disponibilidade do entrevistado/empresa em comprometer seu tempo, para o período de negociação com o entrevistado/empresa e para a logística disponível à aplicação do protocolo de pesquisa em campo.

Para adequar o processo de entrevistas e consequentemente o processo de coleta de dados, algumas decisões foram tomadas no sentido de:

- Tornar a abordagem junto aos prováveis entrevistados mais objetiva;

- Realizar as duas etapas das entrevistas num mesmo encontro com o entrevistado;

- Em alguns casos os entrevistados pediram o envio dos questionários antes da reunião para que o processo fosse "adiantado". Mas esse ponto foi modificado no caso em que os entrevistados tinham o domínio do assunto da pesquisa; e

- Deve-se ter uma agenda flexível e muito organizada para conseguir atender à demanda das entrevistas e não prejudicar o cronograma de atividades.

Outro ponto que deve ser levado em consideração no momento da abordagem para a realização da pesquisa de campo é o de valorizar a participação da empresa e entrevistados no estudo que será feito. Quais os benefícios que a organização poderá obter participando da pesquisa, ou seja, de que forma a pesquisa poderá auxiliar a empresa. Além de tornar visivel a todos os envolvidos a importância do trabalho realizado enquanto pesquisa, da contribuição para a geração de teoria.

\section{Conclusão}

Para que o emprego de $A M T$ possa, de fato, proporcionar vantagem competitiva às empresas, faz-se necessário que a tecnologia seja selecionada segundo critérios que considerem aspectos estratégicos e a sua implantação seja planejada e acompanhada. Como resultado deste processo de "refinamentos sucessivos", obteve-se um conjunto de recomendações para a revisão do projeto organizacional que podem influenciar o processo de adoção de $A M T$. Essas recomendações seriam, na realidade, um conjunto de decisões a ser tomado a partir de várias perspectivas, de tal forma a definir ou conduzir os impactos da adoção da tecnologia na organização.

0 que se tem verificado com frequência é que as empresas dão muito mais importância às questões de cunho financeiro na hora de planejar a implementação de um $A M T$, deixando um pouco de lado outras questões como os impactos na organização, ajuste na estrutura organizacional, capacitação de pessoal, entre outros aspectos que são de relevância nesse processo. A análise prévia do impacto da adoção e implantação de um $A M T$ nas características organizacionais auxilia no processo de seleção de $A M T$.

0 trabalho de Woodward (1977) é um marco de referência de suma importância para o desenvolvimento deste trabalho, pois ele define que há um imperativo tecnológico: a tecnologia adotada pela empresa determina a sua estrutura e comportamento organizacional, e essa foi uma das inspirações para a pesquisa desenvolvida.

\section{Referências}

ABDEL-KADER, M.; LUTHER, R. The impact of firm characteristics on management accounting practices: AUK-based empirical analysis. The British Accounting Review, v. 40, n. 1, p. 2-27, 2008.

BRASIL. Ministério da Ciência e Tecnologia. Estudo da Competitividade da Indústria Brasileira - ECIB. Campinas, 1993. Disponível em: <http://www.mct.gov.br/index.php/ content/view/13637.html>. Acesso em: 01 ago. 2006.

BEATTY, C. Implementing advanced manufacturing technology. Business Quaterly, v. 55, n. 2, p. 46-50, 1990.

BESSANT, J. Towards total integrated manufacturing. International Journal of Production Economics, v. 34, n. 3, p. 237-251, 1994.

BROWN, S.; BLACKMON, K. Aligning manufacturing strategy and business-level competitive strategy in new competitive environments: The case for strategic resonance. Journal of Management Studies, v. 42, n. 4, p. 793-815, 2005.

BURCHER, P.; LEE, G.; SOHAL, A. Lessons for implementing AMT: some case experiences with CNC in Australia, Britain and Canada. International Journal of Operations \& Production Management, v. 19 , n. 5/6, p. 515- 526, 1999. 
BURGELMAN, R. A.; MAIDIQUE, M. A.; WHEELWRIGHT, S. C. Strategic management of technology and innovation. 3. ed. New York: McGraw-Hill//rwin, 2001.

CAMARGO, R. A. F.; VANALLE, R. M. Alinhamento de estratégias: a combinação das inovações tecnológicas e organizacionais. Revista Ciência e Tecnologia, v. 11, n. 20, p. 39-48, 2002.

CARDOSO, R. R.; GOUVÊA DA COSTA, S. E.; PINHEIRO DE LIMA, E. Um estudo das 'barreiras' organizacionais à introdução de novas tecnologias. In: ENCONTRO NACIONAL DE ENGENHARIA DE PRODUÇÃO, 24., Florianópolis, 2004. Anais... Florianópolis: UFSC, 2004

CASTRILLÓN, 1. D.; CANTORNA, A. I.S. The effect of the implementation of advanced manufacturing technologies on training in the manufacturing sector. Journal of European Industrial Training, v. 29, n. 4, p. 268-280, 2005.

CHURCHMAN, C. W. Introdução à teoria de sistemas. Petrópolis: Vozes, 1972

DANGAYACH, G. S.; DESHMUKH, S. G. Advanced manufacturing technology implementation: Evidence from Indian small and medium enterprises SMEs. Journal of Manufacturing Technology Management, v. 16, n. 5, p. 483-496, 2005.

DANGAYACH, G. S.; DESHMUKH, S. G. An exploratory study of manufacturing strategy practices of machinery manufacturing companies in India. OMEGA International Journal of Management Science, v. 34, n. 3, p. 254-273, 2006.

DAS, A.; NARASIMHAN, R. Process-technology fit and its implications for manufacturing performance. Journal of Operations Management, v. 19, n. 5, p. 521-540, 2001.

DAVENPORT, T. H. Putting the enterprise into the enterprise system. Harvard Business Review, v. 76, n. 4, p. 121-131, 1998.

DíAZ, M. S.; MACHUCA, J. A. D.; ÁlVAREZ-GIL, M. J. A view of developing patterns of investment in $A M T$ through empirical taxonomies: new evidence. Journal of Operations Management, v. 21 , n. 5, p. 577-606, 2003.

DREJER, A. Frameworks for the Management of Technology: towards a contingent approach. Technology Analysis \& Strategic Management, v. 8, n. 1, p. 9-12, 1996.

DUIMERING, P. R.; SAFAYENI, F.; PURDY, L. Integrated manufacturing: redesign the organization before implementing flexible technology. Sloan Management Review, v. 34, n. 4, p. 47-56, 1993.

EFSTATHIADES, A.; TASSOU, S.; ANTONIOU, A. Strategic planning, transfer and implementation of Advanced Manufacturing Technologies $(A M T)$. Development of an integrated process plan, Technovation, v. 22, n. 4, p. 201-212, 2002.

FEDERACÃO DAS INDÚSTRIAS DO ESTADO DO PARANÁ - FIEP. VIII Sondagem Industrial: a visão de líderes industriais paranaenses, 2003-2004. Curitiba: FIEP-DEC, 2003.

FEDERAÇÃO DAS INDÚSTRIAS DO ESTADO DO PARANÁ FIEP. IX Sondagem Industrial: a visão de líderes industriais paranaenses, 2004-2005. Curitiba: FIEP-DEC, 2004.

FEDERAÇÃO DAS INDÚSTRIAS DO ESTADO DO PARANÁ - FIEP. $X$ Sondagem Industrial: a visão de líderes industriais paranaenses, 2005-2006. Curitiba: FIEP-DEC, 2005.

FEDERAÇÃO DAS INDÚSTRIAS DO ESTADO DO PARANÁ - FIEP. $X I$ Sondagem Industrial: a visão de líderes industriais paranaenses, 2006-2007. Curitiba: FIEP-DEC, 2006.

FEDERACÃO DAS INDÚSTRIAS DO ESTADO DO PARANÁ - FIEP. XII Sondagem Industrial: a visão de líderes industriais paranaenses, 2007-2008. Curitiba: FIEP-DEC, 2007.

GARRE, M. F. M.; YEPES, J. A. L. Hacia un nuevo modelo de empresa? ESIC Market, n. 90, 1995.

GHANI, K. A.; JAYABALAN, V.; SUGUMAR, M. Impact of advanced manufacturing technology on organizational structure. The Journal of High Technology Management Research, v. 13, n. 2, p. 157-175, 2002.

GOUVÊA DA COSTA, S. E. Uma racionalidade e um processo para a adoção estratégica de tecnologias avançadas de manufatura - AMT. Curitiba: Pontifícia Universidade Católica do Paraná, 2004. 154 p. Trabalho Acadêmico.
GOUVÊA DA COSTA, S. E.; PINHEIRO DE LIMA, E. Advanced manufacturing technology adoption: an integrated approach. Journal of Manufacturing Technology Management, v. 20, n. 1, 2009. p. 74-96.

GOUVÊA DA COSTA, S. E.; PLATTS, K. W.; FLEURY, A. C. C. Strategic selection of advanced manufacturing technologies $(A M T)$, based on the manufacturing vision. International Journal of Computer Applications in Technology, v. 27, n. 1, p. 12-23, 2006.

GUPTA, A.; CHEN, I. J; CHIANG, D. Determining organizational structure choices in advanced manufacturing technology management. OMEGA International Journal of Management Science, v. 25, n. 5, p. 511-521, 1997.

GUPTA, Y. P. Organizational issues of flexible manufacturing systems. Technovation, v. 8, n. 4, p. 255-269, 1988.

GUPTA, Y. P.; LONIAL, S. C. Exploring linkages between manufacturing strategy, business strategy, and organizational strategy. Production and Operations Management, v. 7, n. 3, 1998.

HOFMANN, C.; ORR, S. Advanced manufacturing technology adoption - the German experience. Technovation, v. 25, n. 7, p. 711-724, 2005.

HUTCHISON, J.; DAS, S. R. Examining a firm's decisions with a contingency framework for manufacturing flexibility. International Journal of Operations \& Production Management, v. 27, n. 2, p. 159-180, 2007.

JONES, 0.; SMITH, D. Strategic technology management in a mid-corporate firm: the case of otter controls. Journal of Management Studies, v. 34, n. 4, p. 511-536, 1997.

KHAZANCHI, S.; LEWIS, M. W.; BOYER, K. K. Innovation-supportive culture: The impact of organizational values on process innovation. Journal of Operations Management, v. 25, n. 4, p. 871-884, 2007.

LEWIS, M. W.; BOYER, K. K. Factors impacting AMTimplementation: an integrative and controlled study. Journal of Engineering and Technology Management, v. 19, p. 111-130, 2002.

LIKER, J. K.; MAJCHRZAK, A.; CHOI, T. Impacts of programmable manufacturing technology: a review of recent studies and contingency formulation. Journal of Engineering \& Technology Management, v. 10, p. 229-64, 1993.

LIN, C.; TAN, B.; CHANG, S. The critical factors for technology absorptive capacity. Industrial Management \& Data Systems, v. 102, n. 6, p. 300-308, 2002.

MACDOUGALL, S. L.; PIKE, R. H. Consider your options: changes to strategic value during implementation of advanced manufacturing technology. OMEGA International Journal of Management Science, v. 31, n. 1, p. 1-15, 2003.

MAFFEl, M. J.; MEREDITH, J. The organizational side of flexible manufacturing technology - guidelines for managers. International Journal of Operation \& Production Management, v. 14, n. 8, p. 17-34, 1994.

MARAVIESKI, E. L.; REIS, D. Avaliação de resistência à mudança em processos de inovação: a construção de um instrumento de pesquisa. In: ENCONTRO NACIONAL DE ENGENHARIA DE PRODUCÃO, 28., Rio de Janeiro, 2008. Anais... Rio de Janeiro: ABEPRO, 2008.

MARQUES, A. L.; BORGES, R. S. G.; CARVALHO-FREITAS, M. N. Mudança organizacional: interferência nos indicadores de comprometimento e satisfação no trabalho. In: INTERNATIONAL CONFERENCE OF THE IBEROAMERICAN ACADEMY OF MANAGEMENT, 5., 2007, Santo Domingo. Anais... lberoamerican Academy of Management, 2007.

MARQUES, A. L.; CHAVES, R. C.; DIAS, A. T. Validando um instrumento de medida de resistência à mudança. In: ENANPAD - ENCONTRO DA ANPAD, 29., 2005, Brasília, DF. Anais.... Rio de Janeiro: ANPAD, 2005. v. 29, p. 445.

MENG, Y. C. et al. The impact of organisational characteristics on the outcome of new product development projects in singapore-based firms. Singapore Management Review, v. 20, n. 1, p. 25-42, 1998. 
MEREDITH, J.; VINEYARD, M. A longitudinal study of the role of manufacturing technology in business strategy. International Journal of Operations \& Production Management, v. 13, n. 12, p. 4-24, 1993.

MONGE, C. A. M. et al. Performance measurement of AMT: a cross-regional study. Benchmarking: An International Journal, v. 13, n. 1/2, p. 135-146, 2006.

MULLARKEY, S.; JACKSON, P. R.; PARKER, S. K. Employee reactions to JIT manufacturing practices: a two-phase investigation. International Journal of Operations \& Production Management, v. 15, n. 11, p. 62-79, 1995.

NEMETZ, P. L.; FRY, L. W. Flexible manufacturing organizations: implications for strategy formulation and organizations design. Academy of Management Review, v. 13, n. 4, p. 627-638, 1988.

PANDZA, K.; POLAJNAR, A.; BUCHMEISTER, B. Strategic management of advanced manufacturing technology. The International Journal of Advanced Manufacturing Technology, v. 25 , n. $3 / 4$, p. $402-408,2005$.

PINHEIRO DE LIMA, E.; LEZANA, A. G. R. Desenvolvendo um framework para estudar a ação organizacional: das competências ao modelo organizacional. Gestão \& Produção, v. 12 , n. 2, p. 177-190, 2005.

PINJALA, S. K.; PINTELON, L.; VEREECKE, A. An empirical investigation on the relationship between business and maintenance strategies. International Journal of Production Economics, v. 104, n. 1, p. 214-229, 2006.

PRETORIUS, M. W.; WET, G. A model for assesment of new technology for the manufacturing enterprise. Technovation, v. 20 , n. 1 , p. $3-10,2000$.

RAMAMURTHY, K.; KING, W. R. Computer integrated manufacturing: an exploratory study of key organizational barriers. OMEGA International Journal of Management Science, v. 20, n. 4, p. 475-491, 1992.

RAYMOND, L.; COTREAU, A. M. Enabling the strategic development of SMEs through advanced manufacturing systems: A configurational perspective. Industrial Management \& Data Systems, v. 106, n. 7, p. 1012-1032, 2006.

RAYMOND, L.;St-PIERRE, J. Antecedents and performance outcomes of advanced systems sophistication in SMEs. International Journal of Operations and Production Management, v. 25, n. 6, p. 514-533, 2005.

SÄFSTEN, K.; WINROTH, M.; STAHRE, J. The content and process of automation strategies. International Journal of Production Economics, v. 110, n. 1/2 p. 25-38, 2007.

SALERNO, M. S. Projeto de organizações integradas e flexíveis: processos, grupos e gestão democrática via espaços de comunicação-negociação. São Paulo: Atlas, 1999.

SANCHEZ, C. M.; MCKINLEY, W. Environmental regulatory influence and product innovation: the contingency effects of organizational characteristics. Journal of Engineering and Technology Management, v. 15, n. 4, p. 257-278, 1998.

SHANI, A. B. et al. Advanced manufacturing systems and organizational choice: sociotechnical system approach. California Management Review, v. 34, n. 4, p. 91-111, 1992.

SMALL, M. H. Justifying investment in advanced manufacturing technology: a portfolio analysis. Industrial Management \& Data Systems, v. 106, n. 4, p. 485-508, 2006.

SMALL, M.; YASIN, M. Developing a framework for the effective planning and implementation of advanced manufacturing technology. International Journal of Operations \& Production Management, v. 17, n. 5, p. 468-489, 1997.

SOHAL, A. A longitudinal study of planning and implementation of advanced manufacturing technology. International Journal of Computer Integrated Manufacturing, v. 10, p. 281-95, 1997.

SOHAL, A. Investing in advanced manufacturing technology: comparing Australia and the United Kingdom. Benchmarking for Quality Management \& Technology, v. 1, n. 2, p. 24-41, 1994.

SOHAL, A.; SAMSON, D.; WEILL, P. Manufacturing and technology strategy: a survey of planning for $A M T$, Computer Integrated Manufacturing Systems, v. 4, n. 2, p. 71-79, 1991.

SUN, H. Current and future patterns of using advanced manufacturing technologies. Technovation, v. 20, n. 11, p. 631-641, 2000.

SWINK, M.; NAIR, A. Capturing the competitive advantages of AMT: Design-manufacturing integration as a complementary asset. Journal of Operations Management, v. 25, n. 3, p. 736-754, 2007.

THEODOROU, P.; FLOROU, G. Manufacturing strategies and financial performance - the effect of advanced information technology: CAD/CAM systems. OMEGA International Journal of Management Science, v. 36, n. 1, p. 107-121, 2008.

UNGAN, M. C. Manufacturing best practices: implementation success factors and performance. Journal of Manufacturing Technology Management, v. 18, n. 3, p. 333-348, 2007.

WALDECK, N. E. Worker assessment and the provision of developmental activities with advanced technology: An empirical study. International Journal of Production Economics, v. 107, n. 2, p. 540-554, 2007.

WALDECK, N. E.; LEFFAKIS, W. M. HR perceptions and the provision of workforce training in an $A M T$ environment: An empirical study. OMEGA International Journal of Management Science, v. 35, n. 2, p. 161-172, 2007.

WOODWARD, J. Organização Industrial: teoria e prática. São Paulo: Atlas: 1977.

YANG, J.; DEANE, R. H. Strategic implications of Manufacturing Cell. Integrated Manufacturing Systems, v. 5, n. 4/5, p. 87-96, 1994.

ZHANG, Q.; VONDEREMBSE, M. A.; CAO, M. Achieving flexible manufacturing competence: The roles of advanced manufacturing technology and operations improvement practices. International Journal of Operations \& Production Management, v. 26, n. 6, p. 580-599, 2006.

\title{
Organizational changes in the adoption of advanced manufacturing technologies
}

\begin{abstract}
In order for the adoption of Advanced Manufacturing Technology (AMT) to provide competitive advantages to the companies, it is necessary to select these technologies according to criteria that consider strategic aspects. The organizational characteristics of a company influence particularly the process of $A M T$ adoption, in such a way that it is necessary to develop recommendations for the review of the organizational project. The present work proposes a process for the generation of these recommendations, which through a methodology called 'successive refinements' produces a set of recommendations for the review of the organizational project, based on requirements established by the $A M T$ adoption process. These recommendations are a set of decisions that may be taken about the organizational project to adapt them to demands of the new technology to be introduced, in other words, the $A M T$ implementation.
\end{abstract}

\section{Keywords}

Advanced manufacturing technologies. Organizational change. Manufacturing strategy. Organizational project. 
Apêndice 1. Contribuições da literatura para as dimensões: estrutura, processos e espaços.

\begin{tabular}{|c|c|c|c|}
\hline \multirow{2}{*}{ Dimensão } & \multirow{2}{*}{\begin{tabular}{|c|} 
Problema / Recomendação \\
Definição
\end{tabular}} & \multirow{2}{*}{\multicolumn{2}{|c|}{ Trabalhos relacionados }} \\
\hline & & & \\
\hline & $\begin{array}{l}\text { Grupo de coordenação } \\
\text { Definição de um grupo de coordenação } \\
\text { para gerir o processo de seleção/adoção/ } \\
\text { implementação de } A M T\end{array}$ & $\begin{array}{c}\text { Ungan (2007) } \\
\text { Pinjala, Pintelon e Vereecke (2006) } \\
\text { Cardoso, Gouvêa da Costa e } \\
\text { Pinheiro de Lima (2004) } \\
\text { Meng et al. (1998) } \\
\text { Jones e Smith (1997) }\end{array}$ & $\begin{array}{c}\text { Garre e Yepes (1995) } \\
\text { Sohal (1994) } \\
\text { Duimering, Safayeni e Purdy (1993) } \\
\text { Beatty (1990) } \\
\text { Gupta (1988) }\end{array}$ \\
\hline & $\begin{array}{l}\text { Times de trabalho } \\
\text { Criação de times de trabalho ao longo } \\
\text { do processo }\end{array}$ & $\begin{array}{c}\text { Waldeck (2007) } \\
\text { Waldeck e Leffakis (2007) } \\
\text { Pinjala, Pintelon e Vereecke (2006) } \\
\text { Cardoso, Gouvêa da Costa e } \\
\text { Pinheiro de Lima (2004) } \\
\text { Das e Narasimhan (2001) } \\
\text { Meng et al. (1998) } \\
\text { Gupta, Chen e Chiang (1997) } \\
\text { Garre e Yepes (1995) }\end{array}$ & $\begin{array}{c}\text { Mullarkey, Jackson e Parker (1995) } \\
\text { Sohal (1994) } \\
\text { Duimering, Safayeni e Purdy (1993) } \\
\text { Ramamurthy e King (1992) } \\
\text { Sohal, Samson e Weil (1991) } \\
\text { Beatty (1990) } \\
\text { Nemetz e Fry (1988) } \\
\text { Gupta (1988) }\end{array}$ \\
\hline & $\begin{array}{l}\text { ldentificação de pessoas-chave } \\
\text { Para gerir as informações durante } \\
\text { o processo de seleção/adoção/ } \\
\text { implementação e pós-implementação } \\
\text { de } A M T\end{array}$ & $\begin{array}{c}\text { Ungan (2007) } \\
\text { Khazanchi, Lewis e Boyer (2007) } \\
\text { Hofmann e Orr (2005) }\end{array}$ & $\begin{array}{c}\text { Cardoso, Gouvêa da Costa e } \\
\text { Pinheiro de Lima (2004) } \\
\text { Burcher, Lee e Sohal (1999) } \\
\text { Mullarkey, Jackson e Parker (1995) }\end{array}$ \\
\hline & $\begin{array}{l}\text { Programas de capacitação } \\
\text { Realizar programas de capacitação para } \\
\text { todos os níveis relacionados à } \\
\text { introdução do } A M T\end{array}$ & $\begin{array}{l}\text { Theodorou e Florou (2008) } \\
\text { Waldeck e Leffakis (2007) } \\
\text { Waldeck (2007) } \\
\text { Raymond e St-Pierre (2005) } \\
\text { Pandza, Polajnar e Buchmeister (2005) } \\
\text { Castrillón e Cantorna (2005) } \\
\text { Cardoso, Gouvêa da Costa e } \\
\text { Pinheiro de Lima (2004) }\end{array}$ & $\begin{array}{c}\text { Lewis and Boyer (2002) } \\
\text { Lin, Tan e Chang (2002) } \\
\text { Das e Narasimhan (2001) } \\
\text { Gupta, Chen e Chiang (1997) } \\
\text { Mullarkey, Jackson e Parker (1995) } \\
\text { Meredith e Vineyard (1993) } \\
\text { Ramamurthy e King (1992) }\end{array}$ \\
\hline & $\begin{array}{l}\text { Flexibilidade da organização } \\
\text { Fazer com que a organização fique o mais } \\
\text { flexível possível com relação à introdução } \\
\text { do } A M T\end{array}$ & $\begin{array}{c}\text { Cardoso, Gouvêa da Costa e } \\
\text { Pinheiro de Lima (2004) } \\
\text { Díaz, Machuca e Álvarez-Gil (2003) } \\
\text { Lewis e Boyer (2002) } \\
\text { Meng et al. (1998) } \\
\text { Davenport (1998) } \\
\text { Gupta, Chen e Chiang (1997) }\end{array}$ & $\begin{array}{c}\text { Garre e Yepes (1995) } \\
\text { Maffei e Meredith (1994) } \\
\text { Duimering, Safayeni e Purdy (1993) } \\
\text { Shani et al. (1992) } \\
\text { Nemetz e Fry (1988) }\end{array}$ \\
\hline & $\begin{array}{l}\text { Garantir o alinhamento estratégico } \\
\text { durante o processo }\end{array}$ & $\begin{array}{c}\text { Theodorou e Florou (2008) } \\
\text { Säfsten, Winroth e Stahre (2007) } \\
\text { Swink e Nair (2007) } \\
\text { Monge et al. (2006) } \\
\text { Raymond e St-Pierre (2005) } \\
\text { Pandza, Polajnar e Buchmeister (2005) } \\
\text { Macdougall e Pike (2003) } \\
\text { Díaz, Machuca e Álvarez-Gil (2003) } \\
\text { Efstathiades, Tassou e Antoniou (2002) } \\
\text { Camargo e Vanalle (2002) } \\
\text { Ghani, Jayabalan e Sugumar (2002) } \\
\text { Burgelman, Maidique e Wheelwright (2001) } \\
\text { Das e Narasimhan (2001) } \\
\text { Burcher, Lee e Sohal (1999) }\end{array}$ & $\begin{array}{c}\text { Gupta e Lonial (1998) } \\
\text { Bessant (1994) } \\
\text { Sohal (1997) } \\
\text { Small e Yasin (1997) } \\
\text { Jones e Smith (1997) } \\
\text { Drejer (1996) } \\
\text { Yang e Deane (1994) } \\
\text { Duimering, Safayeni e Purdy (1993) } \\
\text { Meredith e Vineyard (1993) } \\
\text { Shani et al. (1992) } \\
\text { Ramamurthy e King (1992) } \\
\text { Sohal, Samson e Weil (1991) } \\
\text { Beatty (1990) }\end{array}$ \\
\hline & $\begin{array}{l}\text { Regulação do ambiente } x \text { inovação } \\
\text { tecnológica }\end{array}$ & $\begin{array}{c}\text { Waldeck e Leffakis (2007) } \\
\text { Ungan (2007) } \\
\text { Hutchison e Das (2007) } \\
\text { Raymond e Cotreau (2006) }\end{array}$ & $\begin{array}{l}\text { Brown e Blackmon (2005) } \\
\text { Lin, Tan e Chang (2002) } \\
\text { Lewis e Boyer (2002) } \\
\text { Sanchez e McKinley (1998) }\end{array}$ \\
\hline Processos & $\begin{array}{l}\text { Acompanhamento contínuo } \\
\text { Realizar um acompanhamento contínuo } \\
\text { ao longo do processo }\end{array}$ & $\begin{array}{l}\text { Khazanchi, Lewis e Boyer (2007) } \\
\text { Cardoso, Gouvêa da Costa e } \\
\text { Pinheiro de lima (2004) } \\
\text { Efstathiades, Tassou e Antoniou (2002) } \\
\text { Castrillón e Cantorna (2002) }\end{array}$ & $\begin{array}{l}\text { Burgelman, Maidique e } \\
\text { Wheelwright (2001) } \\
\text { Sun (2000) } \\
\text { Sanchez e McKinley (1998) } \\
\text { Garre e Yepes (1995) }\end{array}$ \\
\hline
\end{tabular}


Apêndice 1. Contribuições da literatura para as dimensões: estrutura, processos e espaços.

\begin{tabular}{|c|c|c|c|}
\hline \multirow{2}{*}{ Dimensão } & Problema / Recomendação & \multirow{2}{*}{\multicolumn{2}{|c|}{ Trabalhos relacionados }} \\
\hline & Definição & & \\
\hline \multirow[t]{4}{*}{ Processos } & $\begin{array}{l}\text { Implicações da tecnologia } \\
\text { A organização deve ter um claro } \\
\text { entendimento das implicações da } \\
\text { tecnologia }\end{array}$ & $\begin{array}{c}\text { Small (2006) } \\
\text { Castrillón e Cantorna (2005) } \\
\text { Raymond e St-Pierre (2005) } \\
\text { Cardoso, Gouvêa da Costa e } \\
\text { Pinheiro de Lima (2004) } \\
\text { Lewis e Boyer (2002) } \\
\text { Ghani, Jayabalan e Sugumar (2002) } \\
\text { Efstathiades, Tassou e Antoniou (2002) } \\
\text { Camargo e Vanalle (2002) } \\
\text { Burgelman, Maidique e Wheelwright (2001) } \\
\text { Sun (2000) } \\
\text { Pretorius e Wet (2000) }\end{array}$ & $\begin{array}{c}\text { Davenport (1998) } \\
\text { Sohal (1997) } \\
\text { Small e Yasin (1997) } \\
\text { Gupta, Chen e Chiang (1997) } \\
\text { Drejer (1996) } \\
\text { Yang e Deane (1994) } \\
\text { Sohal (1994) } \\
\text { Meredith e Vineyard (1993) } \\
\text { Duimering, Safayeni e Purdy (1993) } \\
\text { Sohal, Samson e Weil (1991) } \\
\text { Gupta (1988) }\end{array}$ \\
\hline & $\begin{array}{l}\text { Feedback de informações } \\
\text { Manter um feedback constante de } \\
\text { informação por parte dos envolvidos com } \\
\text { relação ao grupo de coordenação } \\
\text { e vice-versa }\end{array}$ & $\begin{array}{c}\text { Theodorou e Florou (2008) } \\
\text { Abdel-Kader e Luther (2008) } \\
\text { Pandza, Polajnar e Buchmeister (2005) } \\
\text { Cardoso, Gouvêa da Costa e } \\
\text { Pinheiro de Lima (2004) }\end{array}$ & $\begin{array}{c}\text { Jones e Smith (1997) } \\
\text { Gupta, Chen e Chiang (1997) } \\
\text { Drejer (1996) }\end{array}$ \\
\hline & $\begin{array}{l}\text { Modificação de processos e infraestrutura } \\
\text { Modificar processos e infraestrutura } \\
\text { organizacionais na preparação para a } \\
\text { adoção do } A M T\end{array}$ & $\begin{array}{c}\text { Waldeck (2007) } \\
\text { Säfsten, Winroth e Stahre (2007) } \\
\text { Raymond e St-Pierre (2005) } \\
\text { Macdougall e Pike (2003) } \\
\text { Díaz, Machuca e Álvarez-Gil (2003) } \\
\text { Ghani, Jayabalan e Sugumar (2002) } \\
\text { Das e Narasimhan (2001) } \\
\end{array}$ & $\begin{array}{c}\text { Salerno (1999) } \\
\text { Small e Yasin (1997) } \\
\text { Jones e Smith(1997) } \\
\text { Bessant (1994) } \\
\text { Yang e Deane (1994) } \\
\text { Duimering, Safayeni e Purdy (1993) } \\
\text { Ramamurthy e King (1992) } \\
\end{array}$ \\
\hline & $\begin{array}{l}\text { Pontos potenciais a modificações } \\
\text { Definir pontos potenciais a modificações } \\
\text { para melhor se adaptar aos requisitos } \\
\text { necessários. 1dentificando as dimensões } \\
\text { chave para a empresa }\end{array}$ & $\begin{array}{c}\text { Dangayach e Deshmukh (2005, 2006) } \\
\text { Brown e Blackmon (2005) } \\
\text { Castrillón e Cantorna (2005) } \\
\text { Cardoso, Gouvêa da Costa e } \\
\text { Pinheiro de Lima (2004) } \\
\text { Lin, Tan e Chang (2002) } \\
\text { Efstathiades, Tassou e Antoniou (2002) } \\
\text { Salerno (1999) } \\
\text { Gupta e Lonial (1998) } \\
\end{array}$ & $\begin{array}{c}\text { Drejer (1996) } \\
\text { Sohal (1994) } \\
\text { Meredith e Vineyard (1993) } \\
\text { Shani et al. (1992) } \\
\text { Ramamurthy e King (1992) } \\
\text { Sohal, Samson e Weil (1991) } \\
\text { Nemetz e Fry (1988) } \\
\text { Gupta (1988) }\end{array}$ \\
\hline \multirow[t]{6}{*}{ Espaços } & $\begin{array}{l}\text { Acompanhamento da } 1^{\text {a }} \text { camada } \\
\text { Na medida do possível, tentar fazer com } \\
\text { que, pelo menos, a primeira camada da } \\
\text { rede acompanhe o processo de transição }\end{array}$ & $\begin{array}{l}\text { Dangayach e Deshmukh }(2005,2006) \\
\text { Cardoso, Gouvêa da Costa e } \\
\text { Pinheiro de Lima (2004) }\end{array}$ & $\begin{array}{l}\text { Pretorius e Wet (2000) } \\
\text { Davenport (1998) }\end{array}$ \\
\hline & $\begin{array}{l}\text { Auditoria pós-implementação } \\
\text { Realizar um processo de auditoria } \\
\text { pós-implementação }\end{array}$ & $\begin{array}{c}\text { Ungan (2007) } \\
\text { Castrillón e Cantorna (2005) } \\
\text { Lewis e Boyer (2002) } \\
\text { Efstathiades, Tassou e Antoniou (2002) }\end{array}$ & $\begin{array}{c}\text { Camargo e Vanalle (2002) } \\
\text { Small e Yasin (1997) } \\
\text { Likerm, Majchrzak e Choi (1993) }\end{array}$ \\
\hline & $\begin{array}{l}\text { Criação de um meio de comunicação } \\
\text { Criação de um meio de comunicação } \\
\text { viável para que haja um total intercâmbio } \\
\text { de informações }\end{array}$ & $\begin{array}{c}\text { Theodorou e Florou (2008) } \\
\text { Khazanchi, Lewis e Boyer (2007) } \\
\text { Cardoso, Gouvêa da Costa e } \\
\text { Pinheiro de Lima (2004) } \\
\text { Lin, Tan e Chang (2002) } \\
\text { Lewis e Boyer (2002) } \\
\text { Ghani, Jayabalan e Sugumar (2002) } \\
\text { Davenport (1998) }\end{array}$ & $\begin{array}{c}\text { Jones e Smith (1997) } \\
\text { Gupta, Chen e Chiang (1997) } \\
\text { Garre e Yepes (1995) } \\
\text { Sohal (1994) } \\
\text { Bessant (1994) } \\
\text { Duimering, Safayeni e Purdy (1993) } \\
\text { Gupta (1988) }\end{array}$ \\
\hline & $\begin{array}{l}\text { Adequação ao mercado-alvo } \\
\text { Adequação total ao mercado-alvo do } \\
\text { produto }\end{array}$ & $\begin{array}{l}\text { Abdel-Kader e Luther (2008) } \\
\text { Brown e Blackmon (2005) } \\
\text { Cardoso, Gouvêa da Costa e } \\
\text { Pinheiro de Lima (2004) } \\
\text { Pretorius e Wet (2000) } \\
\end{array}$ & $\begin{array}{l}\text { Gupta e Lonial (1998) } \\
\text { Sohal (1994) } \\
\text { Meredith e Vineyard (1993) } \\
\text { Ramamurthy e King (1992) }\end{array}$ \\
\hline & $\begin{array}{l}\text { Importância de cada um no todo } \\
\text { Mostrar a importância de cada um para } \\
\text { que o todo funcione da melhor maneira }\end{array}$ & $\begin{array}{c}\text { Hutchison e Das (2007) } \\
\text { Raymond e Cotreau (2006) } \\
\text { Cardoso, Gouvêa da Costa e Pinheiro de } \\
\text { Lima (2004) } \\
\text { Sun (2000) } \\
\text { Davenport (1998) }\end{array}$ & $\begin{array}{c}\text { Sohal (1997) } \\
\text { Mullarkey, Jackson e Parker (1995) } \\
\text { Garre e Yepes (1995) } \\
\text { Bessant (1994) } \\
\text { Ramamurthy e King (1992) }\end{array}$ \\
\hline & $\begin{array}{l}\text { Gerar um cenário } \\
\text { Gerar um cenário para o processo de } \\
\text { cultura/feedback baseado na experiência e } \\
\text { aprendizado, para uma posterior melhoria } \\
\text { contínua }\end{array}$ & $\begin{array}{c}\text { Khazanchi, Lewis e Boyer (2007) } \\
\text { Zhang, Vonderembse e Cao (2006) } \\
\text { Sun (2000) }\end{array}$ & $\begin{array}{c}\text { Sohal (1997) } \\
\text { Sohal, Samson e Weil (1991) }\end{array}$ \\
\hline
\end{tabular}

